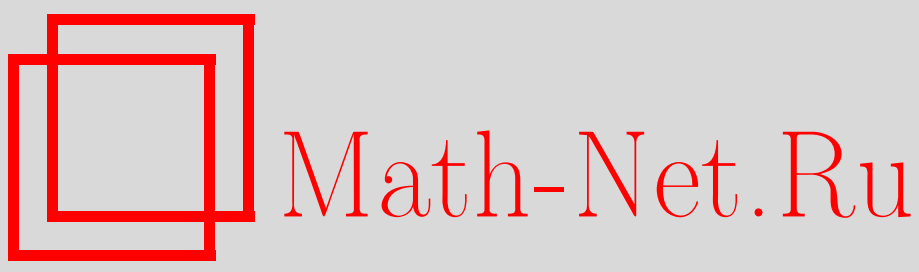

А. Л. Гаркави, В. А. Медведев, С. Я. Хавинсон, О существовании наилучшего равномерного приближения функции нескольких переменных суммой функций меньшего числа переменных, Матем. сб., 1996, том 187, номер 5, 3-14

DOI: https://doi.org/10.4213/sm125

Использование Общероссийского математического портала Math-Net.Ru подразумевает, что вы прочитали и согласны с пользовательским соглашением

http: //www.mathnet.ru/rus/agreement

Параметры загрузки:

IP : 54.205 .225 .156

26 апреля 2023 г., 18:02:38 
УДК 517.5

\author{
А. Л. Гаркави, В.А. Медведев, С.Я. Хавинсон
}

\title{
О существовании наилучшего равномерного приближения функции нескольких переменных суммой функций меньшего числа переменных
}

Пусть заданы отображения $\varphi_{i}$ множества $X$ на множества $X_{i}, i=1, \ldots, n$, $n \geqslant 2$. Вещественная функция $f$ на $X$ приближенно представляется в виде $g_{1} \circ \varphi_{1}+\cdots+g_{n} \circ \varphi_{n}$, где $g_{i}-$ вещественная функция на $X_{i}$. При некоторых ограничениях на отображения $\varphi_{i}$ доказано существование наилучшего равномерного приближения в трех случаях. В первом случае функция $f$ и приближающие суммы ограничены, функции $g_{i}$ могут быть неограниченньпи. Во втором случае функции $f$ и $g_{i}$ ограничены. В третьем случае $f$ и $g_{i}$ непрерьвны, $X$ и $X_{i}-$ метрические компакты, отображения $\varphi_{i}$ непрерьвны.

Библиографофия: 11 названий.

\section{Введение}

В работе изучаются условия проксиминальности подпространств сумм суперпозиций в пространстве ограниченных или непрерывных функций с равномерной метрикой. Напомним, что множество $V$ из метрического пространства $W$ называется проксиминальным, если для $\forall w \in W$ в $V$ сушествует ближайший к $w$ элемент. Через $B(T)$ будем обозначать пространство ограниченных вещественных функций на произвольном множестве $T$; в $B(T)$ определена обычная sup-норма. Изучаемые нами задачи для ограниченных функций состоят в следующем. Заданы произвольные непустые множества $X, X_{1}, \ldots, X_{n}$ и отображения $\varphi_{i}: X \rightarrow X_{i}$, $i=1, \ldots, n, n \geqslant 2$. В пространстве $B(X)$ рассматриваются два подпространства:

$$
\begin{aligned}
& B_{0}(X)=\left\{g_{1} \circ \varphi_{1}+\cdots+g_{n} \circ \varphi_{n}: g_{i} \in B\left(X_{i}\right), i=1, \ldots, n\right\}, \\
& B_{1}(X)=\left\{g_{1} \circ \varphi_{1}+\cdots+g_{n} \circ \varphi_{n}\right\},
\end{aligned}
$$

где $g_{i}$ - произвольные (не обязательно ограниченные) вещественные функции на $X_{i}, i=1, \ldots, n$, но такие, что $g_{1} \circ \varphi_{1}+\cdots+g_{n} \circ \varphi_{n} \in B(X)$. Требуется указать условия, которые надо наложить на множества $X, X_{i}$ и на отображения $\varphi_{i}$, $i=1, \ldots, n$, чтобы подпространство $B_{0}(X)$, соответственно, $B_{1}(X)$ было проксиминальным в $B(X)$.

Перейдем к постановке задачи для непрерьвных функций. Через $C(T)$ будем обозначать пространство непрерывных вещественных функций с равномерной нормой на произвольном компакте $T$ (компактном хаусдорфовом пространстве). Пусть теперь $X, X_{i}$ - компакты, $\varphi_{i}: X \rightarrow X_{i}$ - непрерывные отображения, $i=1, \ldots, n, n \geqslant 2$. В пространстве $C(X)$ рассматривается подпространство

$$
C_{0}(X)=\left\{g_{1} \circ \varphi_{1}+\cdots+g_{n} \circ \varphi_{n}: g_{i} \in C\left(X_{i}\right), i=1, \ldots, n\right\},
$$


и ишутся условия на $X,\left\{X_{i}\right\},\left\{\varphi_{i}\right\}$, чтобы подпространство $C_{0}(X)$ было проксиминальным. Полученные в работе условия проксиминальности $C_{0}(X)$ предполагают, что $X \subset X_{1} \times \cdots \times X_{n}$ или $X \subset X_{1} \times \cdots \times X_{n} \times X_{n+1}$, где $X_{n+1}$ - еше один компакт. Первое включение можно считать выполненным, когда отображение $\varphi=\left(\varphi_{1}, \ldots, \varphi_{n}\right): X \rightarrow X_{1} \times \cdots \times X_{n}$ разделяет точки пространства $X$. В этом случае образ $\varphi(X)$ можно отождествить с $X$, а отображение $\varphi_{i}$ можно истолковать как проектирование из $X$ в $X_{i}$.

Сушествует, однако, естественная ситуация, когда приходится считать, что $X \subset$ $X_{1} \times \cdots \times X_{n+1}$, хотя, по-прежнему, аппроксимация ведется в подпространстве (3), где в устройстве каждой функции из $C_{0}(X)$ задействованы только первые $n$ проекций $\varphi_{i}$. Пусть $X$ - компактное множество в $\mathbb{R}^{p}, p \geqslant 2$. Тогда $C(X)$ состоит из функций $f\left(t_{1}, \ldots, t_{p}\right)$, непрерьвных на $X$. Составим из переменных $t_{1}, \ldots, t_{p}$ наборы $x_{i}=\left(t_{i 1}, \ldots, t_{i p_{i}}\right), i=1, \ldots, n, p_{i}<p, n \geqslant 2$. Считаем, что каждый такой набор не включает в себя целиком никакой другой из них. Пусть $\varphi_{i}$ - отображение, которое каждому $x=\left(t_{1}, \ldots, t_{p}\right) \in X$ ставит в соответствие $x_{i}=\varphi_{i}(x)=\left(t_{i 1}, \ldots, t_{i p_{i}}\right) \in \mathbb{R}^{p_{i}}$, и $X_{i}=\varphi_{i}(X)$. Если любая из переменных $t_{1}, \ldots, t_{p}$ входит хотя бы в один из наборов $x_{i}$, то отображение $\varphi=\left(\varphi_{1}, \ldots, \varphi_{n}\right): X \rightarrow X_{1} \times \cdots \times X_{n}$ есть гомеоморфизм, и мы можем считать, что $X \subset X_{1} \times \cdots \times X_{n}$. Однако, если среди $t_{1}, \ldots, t_{p}$ имеются такие переменные, которые не вошли ни в один набор $x_{i}, i=1, \ldots, n$, то приходится ввести еше один набор $x_{n+1}$, состоящий из всех координат $t_{1}, \ldots, t_{p}$, не вошедших в наборы $x_{i}$, $i=1, \ldots, n$. Тогда отображение $\left(\varphi_{1}, \ldots, \varphi_{n}, \varphi_{n+1}\right): X \rightarrow X_{1} \times \cdots \times X_{n} \times X_{n+1}$ будет гомеоморфизмом, и мы можем считать, что $X \subset X_{1} \times \cdots \times X_{n+1}$, но аппроксимация по-прежнему ведется подпространством (3). В соответствуюшей теореме (теорема 3) мы предусматриваем обе возможности.

История вопроса такова. В работе [1] Дилиберто и Страус рассмотрели задачу равномерной аппроксимации функции $f(x, y)$, непрерывной на прямоугольнике $a \leqslant x \leqslant b, c \leqslant y \leqslant d$, суммами $\varphi(x)+\psi(y)$ с непрерьвньми слагаемыми и доказали, среди других фактов, сушествование наилучшего приближения $\varphi^{*}(x)+\psi^{*}(y)$. (Отметим, что изучаемая в [1] аппроксимационная задача возникла в связи с вопросом экономного размещения информации в небольшой памяти существовавших тогда компьютеров.) В этой работе сделана попытка рассмотрения задачи с большим, чем два, числом переменных, но соответствуюшая теорема сушествования не приводится. Более того, разработанный авторами для случая двух переменных естественный алгоритм последовательного приближения к наилучшей аппроксимирующей сумме, который они "по аналогии" распространяют на произвольное число переменных, оказывается при $n>2$ неверньп [2], [3].

Та же задача аппроксимации функций двух переменных независимо изучалась А.Н. Колмогоровым, В.И. Арнольдом, С.А. Смоляком и Ю.П. Офманом. Их результаты изложены в работе [4]. В частности, в [4] содержится доказанная А.Н. Колмогоровым теорема сушествования, совпадающая с теоремой существования из [1]. Дано также обобщение этой теоремы на функции $n$ переменных в параллелепипеде и начато исследование аппроксимации $f(x, y)$ не на прямоугольнике, а на некотором множестве $X$, лежашем в прямоугольнике. Эта задача сушественно сложнее, так как геометрия $X$ оказывает решающее воздействие на наличие или отсутствие свойства проксиминальности у 
подпространства $C_{0}(X)=\{\varphi(x)+\psi(y)\}$ в $C(X)$. Для выяснения условий, обеспечивающих проксиминальность $C_{0}(X)$, в [4] введены геометрические понятия креста и планки. Горизонтальная планка множества $X$ - это такое сечение $X$ прямой $y=y_{0}$, что проекция этого сечения на ось $O x$ совпадает с проекцией всего множества $X$. Аналогично определяется вертикальная планка. Конечно, планки у $X$ не обязательно сушествуют. Если же обе планки существуют, то их объединение дает крест. В [4] сделана попытка доказать, что если $X$ имеет крест, то всякая функция $f(x, y)$ на $X$, удовлетворяюшая условию Липшица, имеет наилучшее приближение $\varphi^{*}(x)+\psi^{*}(y)$, причем $\varphi^{*}$ и $\psi^{*}$ также удовлетворяют условию Липшица. Приводится и $n$-мерная версия этой теоремы, использующая соответствующее обобщение понятий планок и креста.

Как отметил В.П. Моторный [5], [6], наличие креста не обеспечивает справедливости сформулированного факта. В [5] и [6] это условие заменено другим, более жестким. Последнее также использует наличие крестов, но уже "локальных". В свою очередь, в работах [5], [6] имеется ряд неточностей, связанных, в частности, с тем, что автор не делает различия между подпространствами типа (1) и типа (2). В связи с этим в [7] был приведен пример компакта $X \subset \mathbb{R}^{2}$ и функции $f \in C(X)$, которая не имеет наилучшего приближения ни в $C_{0}(X)$, ни в $B_{0}(X)$, хотя $f(x, y)=\varphi_{0}(x)+\psi_{0}(y)$, где $\varphi_{0}$ и $\psi_{0}$ непрерывны во всех точках $X$, кроме одной (и неограничены). Отображения $\varphi_{1}, \varphi_{2}$ в указанном примере являются проектированиями на оси координат. В работе [8] показано, что наличие креста у $X$ гарантирует проксиминальность $B_{0}(X)$ в $B(X)(n=2)$.

$\mathrm{B}$ настоящей работе установлены три теоремы. Теорема 1 содержит геометрические условия, которые обеспечивают проксиминальность $B_{1}(X)$ в $B(X)$, теоремы 2 и 3 излагают условия, обеспечивающие проксиминальность $B_{0}(X)$ в $B(X)$ и $C_{0}(X)$ в $C(X)$, соответственно. В первых двух теоремах топологическая природа основных множеств несушественна: важны лиш их комбинаторно-геометрические свойства. В третьей теореме, трактуюшей о непрерывных функциях, мы предполагаем множества метрическими компактами, а отображения $\varphi_{i}-$ непрерывными. Существует и вариант теоремы 3 , относящийся к произвольным компактным хаусдорфовьм пространствам, но мы его не излагаем, чтобы избежать дополнительных длиннот (здесь вместо метрики следует использовать равномерные структуры в основных множествах $\left.X_{i}\right)$.

Геометрическим инструментом в теоремах 1 и 2 служит понятие “цепей” различных типов. При $n=2$ введенное понятие $r$-цепи совпадает с понятием молнии. Последнее понятие ввел В.И. Арнольд в знаменитой работе [9], в которой была решена тринадцатая проблема Гильберта. Последнее понятие широко использовалось и в работах по аппроксимации функций двух переменных (см., например, [4], [7]). В теореме 3 дополнительно используется еще одно геометрическое понятие: понятие $(i, \delta)$-максимального сечения, находящееся в родстве с понятием локальной планки. Возможно использование обобщений понятия планки и для получения условий проксиминальности $B_{0}(X)$ в $B(X)$, отличных от условий теоремы 2 , причем упомянутые условия и условия теоремы 2 не содержат одно другого. Результат будет опубликован в другой работе одного из авторов настоящей статьи. 


\section{§1. Случай ограниченных функций}

Введем понятия и обозначения, необходимые для формулировки и доказательства теорем 1 и 2. Определим в $X$ несколько отношений эквивалентности. Точки $x, y$ называются әквивалентными по $r_{i}, i=1, \ldots, n$, если $\varphi_{i}(x)=\varphi_{i}(y)$. Точки $x, y$ называются әквивалентными по $r^{i}, i=2, \ldots, n$, если $\varphi_{j}(x)=\varphi_{j}(y)$ для $j=1, \ldots, i-1$. Отношения $r^{2}$ и $r_{1}$ совпадают. Назовем $r$-цепью длинь $m$ последовательность точек $x^{1}, \ldots, x^{m}$, в которой любые две точки с соседними номерами находятся в одном из отношений $r_{i}$ (возможно, не единственном). Для $i=2, \ldots, n$ назовем $R_{i}$-иепью длины $m$ последовательность точек $x^{1}, \ldots, x^{m}$, в которой любые две точки с соседними номерами находятся в отношении $r_{i}$ или $r^{i}$. Первая и последняя точки цепи любого типа называются концами цепи. Одна точка считается цепью любого типа, концы которой совпадают с этой точкой, а длина равна 1. Будем говорить, что цепь соединяет свои концы. Цеп называется неприводимой, если не сушествует цепи того же типа и с теми же концами, но меньшей длины. В неприводимой цепи все точки различны и каждое последующее отношение отлично от предыдущего. Две точки называются эквивалентными по $R_{i}$ (nо $r$ ), если их можно соединить $R_{i}$-цепю (соответственно, $r$-цепю).

Класс эквивалентности по $R_{i}$ (по $r$ ) назьвается $R_{i}$-классом (r-классом). Если точки находятся в отношении $R_{i}$, то они находятся и в отношении $r$. Поэтому каждый $R_{i}$-класс целиком содержится в некотором $r$-классе. Введенные понятия цепей имеют особенно простой смысл, когда $X \subset \mathbb{R}^{n}, x_{1}, \ldots, x_{n}$ - координаты точки $x=\left(x_{1}, \ldots, x_{n}\right) \in X$, а $\varphi_{i}(x)=x_{i}$, т.е. $\varphi_{i}(x)$ - проекция точки $x$ на $i$-ю координатную ось. Тогда $r$-цеп состоит из вершин ломаной, каждое звено которой параллельно одной из координатных гиперплоскостей. Звенья ломаной не обязательно содержатся в $X$, но вершины принадлежат $X$. Точки $R_{i}$-цепи в таком случае являются вершинами ломаной, каждое звено которой ортогонально $i$-й координатной оси или параллельно координатной плоскости, содержащей оси с номерами $i, i+1, \ldots, n$.

Известно [10], что пространство $B(X)$ сопряжено к пространству $l_{1}(X)$. Следовательно, пространство $l_{1}(X)$ определяет на $B(X)$ слабую $*$-топологию. В тексте будут встречаться линейные функционалы на $B(X)$ вида $L f=\mu_{1} f\left(x^{1}\right)+\cdots+$ $\mu_{s} f\left(x^{s}\right)$, где $x^{1}, \ldots, x^{s}$ - любые фиксированные точки из $X, \mu_{1}, \ldots, \mu_{s}$ - заданные вешественные числа, $f$ - произвольная функция из $B(X)$. Эти функционалы непрерывны в слабой $*$-топологии. В доказательстве теоремы 1 существенно, что $L f$ имеет смысл и в случае, когда $f$ - неограниченная функция. Обозначим через $l_{0}$ множество всех функционалов указанного вида.

ТЕОРема 1. Пусть для $n>2$ каждый $r$-класс содержит конечное число $R_{i}$-классов, $i=3, \ldots, n$. Тогда $B_{1}(X)$ замкнуто в слабой *-топологии $и$, следовательно, каждая функиия из $B(X)$ имеет наилучшее приближение в $B_{1}(X)$. Eсли $n=2$, то заключение теоремы верно без каких-либо ограничений на классъи эквивалентности.

ДоказАтЕльство. Обозначим через $G_{i}$ график отношения $r_{i}, i=1, \ldots, n$, т.е. множество пар $(x, y)$ таких, что $\varphi_{i}(x)=\varphi_{i}(y)$. Рассмотрим сначала случай $n=2$. В каждом $r$-классе $E$ отметим точку $x^{1}=x^{1}(E)$. Произвольную точку $x \in E$ соединим с точкой $x^{1}$ одной неприводимой $r$-цепю $x^{1}, \ldots, x^{m}=x$, которую назовем отмеченной. Пусть $f \in B_{1}(X)$. Запишем $f$ в виде $f=h_{1}+h_{2}$, где функции 
$h_{i}, i=1,2$, удовлетворяют условию: $h_{i}(x)=h_{i}(y)$, если $(x, y) \in G_{i}$. Обозначим $a_{1}=h_{1}\left(x^{1}\right), a_{2}=h_{2}\left(x^{1}\right)$. Точки $x^{p}$ и $x^{p+1}$ находятся в некотором отношении $r_{i_{p}}$, $p=1, \ldots, m-1$. При переходе из точки $x^{p}$ в точку $x^{p+1}$ значение функции $h_{i}$ не изменяется, если $i_{p}=i$. Если $i_{p} \neq i$, то $h_{i}\left(x^{p+1}\right)-h_{i}\left(x^{p}\right)=f\left(x^{p+1}\right)-f\left(x^{p}\right)$, так как $f=h_{1}+h_{2}$. Для каждой точки $x \in X$ имеем:

$$
\begin{aligned}
& h_{1}(x)=a_{1}+\sum_{i_{p}=2}\left(f\left(x^{p+1}\right)-f\left(x^{p}\right)\right), \\
& h_{2}(x)=a_{2}+\sum_{i_{p}=1}\left(f\left(x^{p+1}\right)-f\left(x^{p}\right)\right) .
\end{aligned}
$$

Числа $a_{1}$ и $a_{2}$ зависят от $r$-класса, которому принадлежит $x$. Обозначим через $A_{1 x} f$ и $A_{2 x} f$ суммы, стояшие в правых частях (4) и (5), не включая в них $a_{1}$ и $a_{2}$. Если фиксировать $x$, то $A_{1 x}$ и $A_{2 x}$ - функционалы из $l_{0}$. Для $(x, y) \in G_{i}$ верно равенство $h_{i}(x)=h_{i}(y)$, которое равносильно равенству $\left(A_{i x}-A_{i y}\right) f=0$. Таким образом, если $f \in B_{1}(X)$, то

$$
\left(A_{i x}-A_{i y}\right) f=0, \quad(x, y) \in G_{i}, \quad i=1,2 .
$$

Пусть теперь $f \in B(X)$ и $f$ удовлетворяет равенствам (6). Докажем, что $f \in B_{1}(x)$. Для каждого $r$-класса $E$ выберем числа $a_{1}, a_{2}$ так, чтобы $a_{1}+a_{2}=$ $f\left(x^{1}\right)$, где $x^{1}=x^{1}(E)$. Определим на $X$ две функции $h_{1}$ и $h_{2}$ следуюшим образом. Положим $h_{1}\left(x^{1}\right)=a_{1}, h_{2}\left(x^{1}\right)=a_{2}$. Для остальных точек $x$ значения функций $h_{i}$ зададим равенствами (4), (5), используя в каждом $r$-классе отмеченные цепи. В силу (4), (5) и выбора $a_{1}, a_{2}$ верно равенство $f=h_{1}+h_{2}$. Из (6) следует $h_{i}(x)=h_{i}(y)$, если $(x, y) \in G_{i}$. Поэтому $f \in B_{1}(X)$. Следовательно, $B_{1}(X)$ состоит из тех и только тех функций $f \in B(X)$, которые удовлетворяют равенствам (6). Так как функционалы $A_{i x}-A_{i y}$ непрерывны в слабой *-топологии, то $B_{1}(X)$ замкнуто в этой топологии.

Пусть $n>2$. Предположим сначала, что $X$ состоит из одного $r$-класса. Для

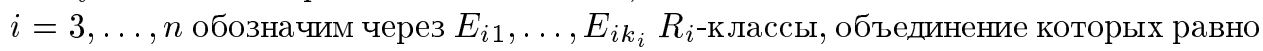
$X$. В каждом классе $E_{i j}$ отметим одну точку $x_{i j}$. Зафиксируем $i$. Если $x \in X$, то $x \in E_{i j}$ при некотором $j$. Соединим $x$ с $x_{i j}$ одной неприводимой $R_{i \text {-цепью }}$ $x^{1}=x_{i j}, x^{2}, \ldots, x^{m}=x$, которую назовем отмеченной. Мы сохраняем обозначения $x^{1}, \ldots, x^{m}$, которые использовались в доказательстве для $n=2$, но теперь точки $x^{p}$ могут быть другими. Обозначим через $A$ множество тех значений $p$, для которых точки $x^{p}$ и $x^{p+1}$ отмеченной цепи находятся в отношении $r^{i}$. Если $m=1$, то $A=\varnothing$. В векторном пространстве $D$ всех вешественных функций $g$ на $X$ определим линейный оператор $A_{i}: D \rightarrow D$, полагая

$$
\left(A_{i} g\right)(x)=\sum_{p \in A}\left(g\left(x^{p+1}\right)-g\left(x^{p}\right)\right) .
$$

Правая часть этого равенства зависит от $x$, поскольку отмеченная $R_{i}$-цепь зависит от $x$. Определим таким способом $A_{i}$ для всех $i=3, \ldots, n$. Для произвольного линейного оператора $L: D \rightarrow D$ и фиксированного $x \in X$ через $L_{x}$ будем обозначать функционал, определяемый равенством $L_{x} f=(L f)(x)$. 
Возьмем функцию $f \in B_{1}(X)$. Запишем ее в виде $f=h_{1}+\cdots+h_{n}$, где функции $h_{i}$ удовлетворяют условию: $h_{i}(x)=h_{i}(y)$, если $(x, y) \in G_{i}$. Обозначим $a_{i j}=h_{i}\left(x_{i j}\right), i=3, \ldots, n, j=1, \ldots, k_{i}$. Если $x \in E_{n j}$, то аналогично (4), (5) получим

$$
h_{n}(x)=a_{n j}+A_{n x} f .
$$

Если $3 \leqslant i<n$ и $x \in E_{i j}$, то

$$
h_{i}(x)=a_{i j}+A_{i x}\left(f-h_{i+1}-\ldots-h_{n}\right) .
$$

Перепишем (7) и (8) в другом виде. Обозначим через $f_{i j}$ характеристическую функцию класса $E_{i j}$. Тогда

$$
\begin{aligned}
h_{n} & =\sum_{j=1}^{k_{n}} a_{n j} f_{n j}+A_{n} f, \\
h_{i} & =\sum_{j=1}^{k_{i}} a_{i j} f_{i j}+A_{i}\left(f-h_{i+1}-\ldots-h_{n}\right),
\end{aligned}
$$

$i=3, \ldots, n$. Для функций $h_{1}$ и $h_{2}$ напишем равенства, аналогичные (10). Для этого повторим с некоторыми изменениями вывод (4), (5). В случае $n=2$ отношения $r$ и $R_{2}$ совпадают. Этого совпадения нет, если $n>2$. Отметим в каждом $R_{2}$-классе $E$ точку $x^{1}=x^{1}(E)$. Каждую точку $x \in E$ соединим с этой точкой $x^{1}$ одной неприводимой $R_{2}$-цепью $x^{1}, \ldots, x^{m}=x$, которую назовем отмеченной. Точки $x^{p}$ и $x^{p+1}$ находятся в некотором отношении $r_{i_{p}}$, где $i_{p}=1$ или $i_{p}=2, p=1, \ldots, m-1$. Определим следующим образом линейные операторы $A_{1}$ и $A_{2}$. Для произвольной функции $g \in D$ положим

$$
\begin{aligned}
& \left(A_{1} g\right)(x)=\sum_{i_{p}=2}\left(g\left(x^{p+1}\right)-g\left(x^{p}\right)\right), \\
& \left(A_{2} g\right)(x)=\sum_{i_{p}=1}\left(g\left(x^{p+1}\right)-g\left(x^{p}\right)\right) .
\end{aligned}
$$

Если сумма не содержит слагаемых, то она считается равной нулю. Определим функции $a_{1}$ и $a_{2}$ на $X$ равенствами $a_{i}(x)=h_{i}\left(x^{1}(E)\right)$, если $x$ принадлежит $R_{2}$-классу $E, i=1,2$. Тогда аналогично (4), (5)

$$
\begin{aligned}
& h_{1}=a_{1}+A_{1}\left(f-h_{3}-\ldots-h_{n}\right), \\
& h_{2}=a_{2}+A_{2}\left(f-h_{3}-\ldots-h_{n}\right) .
\end{aligned}
$$

Подставим выражение (9) для $h_{n}$ в (10) при $i=n-1$. Полученное новое выражение для $h_{n-1}$ и выражение (9) для $h_{n}$ подставим в (10) при $i=n-2$ и т.д. В итоге, для $i=3, \ldots, n$ получим

$$
h_{i}=\sum_{s=3}^{n} \sum_{j=1}^{k_{s}} a_{s j} g_{s j}^{i}+L_{i} f,
$$


где $g_{s j}^{i}$ - функции на $X$, не зависящие от выбора $f, L_{i}$ - линейные операторы. Операторы $L_{i}$ определяются индуктивно равенствами: $L_{n}=A_{n}, L_{i}=A_{i}-$ $A_{i}\left(L_{i+1}+\cdots+L_{n}\right)$. Аналогично $(13)$, получим

$$
\begin{aligned}
& h_{1}=a_{1}+\sum_{s=3}^{n} \sum_{j=1}^{k_{s}} a_{s j} g_{s j}^{1}+L_{1} f, \\
& h_{2}=a_{2}+\sum_{s=3}^{n} \sum_{j=1}^{k_{s}} a_{s j} g_{s j}^{2}+L_{2} f,
\end{aligned}
$$

где $L_{1}=A_{1}-A_{1}\left(L_{3}+\cdots+L_{n}\right), L_{2}=A_{2}-A_{2}\left(L_{3}+\cdots+L_{n}\right)$, функции $g_{s j}^{1}$ и $g_{s j}^{2}$ не зависят от выбора $f$.

Рассмотрим все пары $(x, y) \in G_{i}, i=1, \ldots, n$. Для таких $(x, y)$ верны равенства $h_{i}(x)=h_{i}(y)$. Следовательно,

$$
\sum_{s=3}^{n} \sum_{j=1}^{k_{s}} a_{s j}\left(g_{s j}^{i}(x)-g_{s j}^{i}(y)\right)=\left(L_{i y}-L_{i x}\right) f
$$

если $(x, y) \in G_{i}, i=1, \ldots, n$. Равенства (16) не содержат $a_{1}, a_{2}$, так как функции $a_{1}, a_{2}$ постоянны на $R_{2}$-классах. Будем рассматривать равенства (16) как систему уравнений относительно всех $a_{i j}$. Расположим все $a_{i j}$ в виде строки, которую обозначим через $a$. Тогда $a \in \mathbb{R}^{k}$, где $k=k_{3}+\cdots+k_{n}$. Отметим каждое уравнение (16) индексом $\alpha$ так, чтобы разным уравнением соответствовали разные индексы. По существу, в роли $\alpha$ берется тройка $(i, x, y)$. Система (16) примет вид

$$
\left(b_{\alpha}, a\right)=L_{\alpha} f, \quad \alpha \in I
$$

где $b_{\alpha} \in \mathbb{R}^{k}, L_{\alpha}$ - линейньй функционал. Функционалы $L_{\alpha}$ принадлежат $l_{0}$. Действительно, $A_{i x} \in l_{0}$ для любых $i$ и $x \in X$. Отсюда следует, что $\left(A_{i} A_{j}\right)_{x} \in l_{0}$ для любых $i, j$ и $x \in X$. По индукции легко получить, что $L_{i x}-L_{i y} \in l_{0}$, т.е. $L_{\alpha} \in l_{0}$. Выделим из (17) подсистему с максимальным набором линейно независимых векторов $b_{\alpha}$ :

$$
\left(b_{\alpha}, a\right)=L_{\alpha} f, \quad \alpha \in I_{0} .
$$

Неизвестные $a_{i j}$, коэффициенты при которых в (18) составляют невырожденную матрицу, назовем главньпи. Остальные $a_{i j}$ назовем свободньпи. Если в $(17)$ все $b_{\alpha}$ равны нулю, то все $a_{i j}$ свободные, $I_{0}=\varnothing$. Мы не обсуждаем возможность такого случая, так как это не имеет значения для доказательства теоремы. Выразим из $(18)$ все $a_{i j}$ через свободные неизвестные, используя, например, формулы Крамера. Получим:

$$
a_{i j}=l_{i j} f+l_{i j}^{\prime}(a), \quad i=3, \ldots, n, \quad j=1, \ldots, k_{i},
$$

где $l_{i j} \in l_{0}, l_{i j}^{\prime}(a)$ - линейное выражение от $a$, в котором коэффициенты при главных $a_{i j}$ равны нулю. Для свободных $a_{i j}$ равенства (19) преврашаются, конечно, в тождества $a_{i j}=a_{i j}$. Равенства (17) являются следствием равенств (18). Подставим выражения (19) в равенства (17), не вошедшие в (18). Полученные таким 
способом равенства будут выполняться для произвольных значений свободных неизвестных, т.е. не будут содержать никакого $a_{i j}$. Если перенести правую часть налево, то полученные равенства примут вид

$$
l_{\alpha} f=0, \quad \alpha \in I \backslash I_{0},
$$

где $l_{\alpha} \in l_{0}$.

Пусть теперь $f \in B(X)$ и $f$ удовлетворяет равенствам (20). Докажем, что $f \in B_{1}(X)$. Будем строить функции $h_{i}$ следующим образом. Зададим $a_{i j}$ равенствами (19), приравняв свободные неизвестные к нулю. Для $i=3, \ldots, n$ зададим функции $h_{i}$ равенствами (13). Выберем функции $a_{1}, a_{2}$ так, чтобы они были постоянньми на каждом $R_{2}$-классе $E$ и чтобы выполнялись равенства $a_{1}\left(x^{1}\right)+a_{2}\left(x^{1}\right)=$ $f\left(x^{1}\right)-h_{3}\left(x^{1}\right)-\cdots-h_{n}\left(x^{1}\right)$ для $x^{1}=x^{1}(E)$. Зададим функции $h_{1}, h_{2}$ равенствами (11), (12) или, что то же самое, равенствами $(14),(15)$. В силу (11), (12) и выбора функций $a_{1}, a_{2}$ верно равенство $h_{1}+h_{2}=f-h_{3}-\cdots-h_{n}$. Тогда $f=h_{1}+\cdots+h_{n}$. Из (19) и (20) следует (17). Поэтому верны равенства (16), которые означают, что $h_{i}(x)=h_{i}(y)$ для $(x, y) \in G_{i}$. Следовательно, $f \in B_{1}(X)$. Если $X$ состоит из более чем одного $r$-класса, то равенства (20) для функции $f \in B_{1}(X)$ можно вывести на каждом $r$-классе отдельно. В той части доказательства, где строятся функции $h_{i}$ в предположении, что $f \in B(X)$ и $f$ удовлетворяет равенствам (20), построение $h_{i}$ тоже можно осушествить на каждом $r$-классе отдельно и объединить результаты построения по всем $r$-классам. Опять получится, что $f \in B_{1}(X)$. Следовательно, $B_{1}(X)$ состоит из тех и только тех ограниченных функций $f$, которые удовлетворяют равенствам (20). Так как функционалы $l_{\alpha}$ непрерьвны в слабой *-топологии, то $B_{1}(X)$ замкнуто в этой топологии. Отсюда, в свою очередь, следует, что каждая функция из $B(X)$ имеет наилучшее приближение в $B_{1}(X)$. Теорема доказана.

ТЕОРема 2. Пусть для некоторого натурального числа $N$ длины неприводимых $R_{i}$-иепей не превосходят $N, i=2, \ldots, n$. B случае $n>2$ предположим также, что для $i=3, \ldots, n$ каждый $r$-класс содержит не более $N$ $R_{i}$-классов. Тогда $B_{1}(X)=B_{0}(X)$ и кажсдая функиия из $B(X)$ имеет наилучшее приближение в $B_{0}(X)$.

ДокАЗАТЕЛьСтво. Сушествование наилучшего приближения будет следовать из равенства $B_{1}(X)=B_{0}(X)$ и теоремы 1 . Пусть $n=2$ и $f \in B_{1}(X)$. Тогда $f=h_{1}+h_{2}, h_{i}(x)=h_{i}(y)$, если $\varphi_{i}(x)=\varphi_{i}(y), i=1,2$. Представление $f$ суммой указанного вида не единственно. Возьмем те функции $h_{i}$, построение которых описано сразу после (6). Потребуем дополнительно, чтобы $a_{1}=a_{2}$. Тогда $\left|a_{i}\right| \leqslant \frac{1}{2}|f|$. Отмеченные цепи, использованные при выводе (4) и (5), неприводимые. Поэтому $\left|A_{i x} f\right| \leqslant N|f|, i=1,2$. Тогда $h_{1}$ и $h_{2}$ - ограниченные функции. Следовательно, $B_{1}(X)=B_{0}(X)$.

Пусть $n>2$ и $f \in B_{1}(X)$. Предположим сначала, что $X$ состоит из одного $r$-класса. Как и выше, представление $f$ в виде $h_{1}+\cdots+h_{n}$ не единственно. Возьмем функции $h_{i}$, построение которых описано после (20). Из неприводимости отмеченных цепей и из предположений теоремы следует $\left|A_{i}\right| \leqslant N, i=1, \ldots, n$, если операторы $A_{i}$ рассматривать на $B(X)$. Отсюда и из индуктивного определения операторов $L_{i}$ следует $\left|L_{i}\right| \leqslant N_{1}=N_{1}(n, N)$. Последнее равенство означает, что 
$N_{1}$ можно определить по $n$ и $N$. Характеристические функции $f_{i j}$ имеют значения 0 или 1 . Построение функций $g_{s t}^{q}$ по характеристическим функциям $f_{i j}$ можно осуществить методом индукции. Полное изложение этой индукции излишне. Достаточно заметить, что из оценки $\left|A_{i}\right| \leqslant N$ следует оценка $\left|g_{s t}^{q}\right| \leqslant N_{2}=N_{2}(n, N)$. Кроме того, все значения функций $g_{s t}^{q}$ целые.

Далее, система (17) отличается от (16) только обозначениями. Поэтому $\left|L_{\alpha} f\right| \leqslant$ $2 N_{1}|f|$, а координаты вектора $b_{\alpha} \in \mathbb{R}^{k}$ целые и не превосходят $2 N_{2}$. Тогда базисный определитель системы уравнений (18) по модулю не меньше единицы. Число уравнений в (18) не превосходит $k=k_{3}+\cdots+k_{n} \leqslant(n-2) N$. Нетрудно оценить определители, используемые в формулах Крамера при переходе от (18) к (19) и получить оценку $\left|l_{i j} f\right| \leqslant N_{3}|f|, N_{3}=N_{3}(n, N)$. Так как мы приравняли к нулю свободные неизвестные, то $\left|a_{i j}\right| \leqslant N_{3}|f|$ для всех $a_{i j}$. Для $i=3, \ldots, n$ из (13) получим $\left|h_{i}\right| \leqslant N_{4}|f|, N_{4}=N_{4}(n, N)$. При построении $h_{1}$ и $h_{2}$ дополнительно потребуем, чтобы $a_{1}=a_{2}$. Тогда $\left|a_{i}\right| \leqslant \frac{1}{2}\left|f-h_{3}-\cdots-h_{n}\right|$. В итоге, для всех $i=1, \ldots, n$ получается оценка вида $\left|h_{i}\right| \leqslant N_{5}|f|, N_{5}=N_{5}(n, N)$. Следовательно, $f \in B_{0}(X)$. Если $X$ состоит из более чем одного $r$-класса, то построим функции $h_{i}$ изложенным способом на каждом $r$-классе отдельно, а затем объединим результаты построения. Мы получим функции $h_{i}$, определенные на всем $X$. Они удовлетворяют условиям: $f=h_{1}+\cdots+h_{n}$ и $h_{i}(x)=h_{i}(y)$, если $\varphi_{i}(x)=\varphi_{i}(y)$. Кроме того, функции $h_{i}$ ограничены, поскольку число $N_{5}$ определяется по $n$ и $N$ одинаково для всех $r$-классов. Тогда $f \in B_{0}(X)$. Следовательно, $B_{1}(X)=B_{0}(X)$. Теорема доказана.

\section{§2. Случай непрерывных функций}

Мы предполагаем теперь, что $X$ - компактное подмножество произведения $X_{1} \times \cdots \times X_{n}$ или произведения $X_{1} \times \cdots \times X_{n+1}$, где каждый множитель $X_{i}$ является метрическим компактом. Отображение $\varphi_{i}$ есть проектирование на $X_{i}$. Проектирование $\varphi_{i}$ рассматривается только для точек из $X$. Это следует иметь в виду, когда используется символ $\varphi_{i}^{-1}$. Если $x_{i} \in X_{i}$, то множество $\varphi_{i}^{-1}\left(x_{i}\right)$ называется сечением множества $X$ в точке $x_{i}$. Пусть $F \subset X_{i}$ для некоторого $i \leqslant n$. Будем говорить, что $F$ имеет $i$-максимальное сечение, если существует точка $x_{0 i} \in F$, обладаюшая свойством: для любой точки $y \in \varphi_{i}^{-1}(F)$ найдется точка $x \in \varphi_{i}^{-1}\left(x_{0}\right)$ такая, что $\varphi_{j}(y)=\varphi_{j}(x)$ для $j \neq i$. Другими словами, для каждого $y_{i} \in F$ проекция сечения $\varphi_{i}^{-1}\left(y_{i}\right)$ на произведение всех $X_{j}, j \neq i$, содержится в проекции сечения $\varphi_{i}^{-1}\left(x_{0 i}\right)$ на это же произведение. Пусть $1<i<n$, если $X \subset$ $X_{1} \times \cdots \times X_{n}$, и $1<i \leqslant n$, если $X \subset X_{1} \times \ldots X_{n} \times X_{n+1}$. Зададим $\delta>0$. Будем говорить, что множество $F \subset X_{i}$ имеет $(i, \delta)$-максимальное сечение, если существует точка $x_{0 i} \in F$, обладаюшая свойством: для любой точки $y \in \varphi_{i}^{-1}(F)$ найдется точка $x \in \varphi_{i}^{-1}\left(x_{0 i}\right)$ такая, что $\varphi_{j}(y)=\varphi_{j}(x)$ для $j<i$, и $\rho_{j}\left(\varphi_{j}(y), \varphi_{j}(x)\right)<\delta$ для $j>i$. Здесь $\rho_{j}$ обозначает расстояние между указанными точками в пространстве $X_{j}$. В случае $X \subset X_{1} \times \cdots \times X_{n}$ и $F \subset X_{n}$ будем также говорить, что $F$ имеет $(n, \delta)$-максимальное сечение, если $F$ имеет $n$-максимальное сечение.

Tеорема 3. Пусть для каждого $x_{i} \in X_{i}, i=2, \ldots, n, u$ любого $\delta>0$ найдется положительное $\delta_{0}<\delta$ такое, что замкнутая $\delta_{0}$-окрестность точки $x_{i}$ имеет $(i, \delta)$-максимальное сечение. Тогда каждая функиия из $C(X)$ имеет наилучшее приближение в $C_{0}(X)$, которое одновременно является наилучшим приближсением в $B_{0}(X)$. 
Доказательству теоремы предпошлем следуюшую лемму.

ЛЕмМА. Если выполняется предположсение теоремы 3 , то для всех $i=$ $2, \ldots, n$ число $R_{i}$-классов конечно, и длинь неприводимых $R_{i}$-цепей имеют конечную верхнюю грань.

ДокАЗАТЕЛЬство. Зададим одно из значений $i=2, \ldots, n$. Для каждой точки $x_{i} \in X_{i}$ найдется замкнутый шар с центром в $x_{i}$, который имеет $(i, 1)$-максимальное сечение. Так как $X_{i}$ компактно, то можно выделить конечное число таких шаров $S_{1}, \ldots, S_{q}$, которые покрывают $X_{i}$. Пусть $x_{1 i}, \ldots, x_{q i}$ - точки из $X_{i}$, в которых шары $S_{1}, \ldots, S_{q}$ имеют $(i, 1)$-максимальные сечения. Обозначим $D_{p}=\varphi_{i}^{-1}\left(S_{p}\right)$, $p=1, \ldots, q$. Пусть точки $y, z$ принадлежат некоторому $D_{p}$. Тогда в $X$ найдутся точки $u, v$ такие, что $\varphi_{i}(u)=\varphi_{i}(v)=x_{p i}, \varphi_{j}(u)=\varphi_{j}(y), \varphi_{j}(v)=\varphi_{j}(z)$ для $j=1, \ldots, i-1$. Точки $y, u$ находятся в отношении $r^{i}$. Точки $u, v$ находятся в отношении $r_{i}$. Точки $v, z$ находятся в отношении $r^{i}$. Таким образом, точки $y, z$ соединены $R_{i}$-цепю длины 4 . Следовательно, $D_{p}$ целиком содержится в некотором

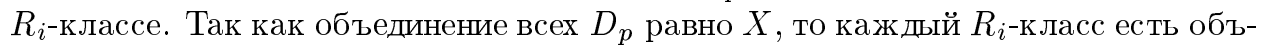
единение некоторых $D_{p}$. Поэтому число $R_{i}$-классов конечно. В каждом множестве $D_{p}$ отметим одну точку $x^{p}$. Если $D_{p}$ и $D_{s}$ содержатся в одном $R_{i}$-классе, то точки $x^{p}$ и $x^{s}$ можно соединить $R_{i}$-цепю некоторой длины $m_{p s}$. Пусть точки $y$ и $z$ эквивалентны по $R_{i}$. Тогда $y \in D_{p}, z \in D_{s}$ для некоторых $p, s$. Если $p=s$, то точки $y$ и $z$ можно соединить, как уже доказано, $R_{i}$-цепюю длины 4 . Если $p \neq s$, то соединим точку $y$ с точкой $x^{p}$, точку $x^{p}$ с точкой $x^{s}$ и точку $x^{s}$ с точкой $z R_{i}$-цепями, длины которых, соответственно, равны $4, m_{p s}$ и 4 . В результате точки $y$ и $z$ будут соединены $R_{i}$-цепњ длины $6+m_{p s}$. Отсюда следует, что длины неприводимых $R_{i}$-цепей не превосходят $6+\max m_{p s}$, где максимум берется по тем парам $p, s$, для которых $D_{p}, D_{s}$ содержатся в одном $R_{i}$-классе. Лемма доказана.

ДокАЗАТЕЛЬСтво ТЕОРЕмЫ 3. Проведем доказательство теоремы для случая $X \subset X_{1} \times \cdots \times X_{n+1}$, которое с незначительными изменениями справедливо и для случая $X \subset X_{1} \times \cdots \times X_{n}$. Пусть $f \in C(X)$. По лемме и по теореме 2 функция $f$ имеет наилучшее приближение $g_{1} \circ \varphi_{1}+\cdots+g_{n} \circ \varphi_{n}$ в $B_{0}(X)$. Применим индукцию. Предположим, что для некоторого $i \geqslant 2$ найдены непрерывные функции $g_{i+1}^{*}, \ldots, g_{n}^{*}$ такие, что сумма

$$
g_{1} \circ \varphi_{1}+\cdots+g_{i} \circ \varphi_{i}+g_{i+1}^{*} \circ \varphi_{i+1}+\cdots+g_{n}^{*} \circ \varphi_{n}
$$

является наилучшим приближением функции $f$ в $B_{0}(X)$. Если $i=n$, то в (21) подразумевается сумма $g_{1} \circ \varphi_{1}+\cdots+g_{n} \circ \varphi_{n}$. Будем искать непрерывную функцию $g_{i}^{*}$ такую, что если в сумме $(21)$ заменить $g_{i}$ на $g_{i}^{*}$, то сумма останется наилучшим приближением функции $f$ в $B_{0}(X)$. Для $x=\left(x_{1}, \ldots, x_{n+1}\right) \in X$ обозначим $h(x)=$ $f(x)-g_{i+1}^{*}\left(x_{i+1}\right)-\cdots-g_{n}^{*}\left(x_{n}\right), g(x)=g_{1}\left(x_{1}\right)+\cdots+g_{i-1}\left(x_{i-1}\right), F(x)=h(x)-g(x)$. Если $i=n$, то $h(x)=f(x)$. Обозначим через $d$ расстояние от $f$ до $B_{0}(X)$. Для всех $x \in X$ имеем

$$
F(x)-d \leqslant g_{i}\left(x_{i}\right) \leqslant F(x)+d
$$

Из (22) следует, что

$$
F(y)-F(z) \leqslant 2 d \text { если } \varphi_{i}(z)=\varphi_{i}(y)
$$


Зафиксируем $x_{i}$ и зададим $\varepsilon>0$. Так как функция $h$ непрерывна, а пространство $X$ компактно, то функция $h$ равномерно непрерывна. Поэтому сушествуют число $\delta>0$ и замкнутый шар $U$ с центром $x_{i}$ в пространстве $X_{i}$ такие, что верно неравенство

$$
|h(u)-h(v)|<\varepsilon,
$$

если точки $u=\left(u_{1}, \ldots, u_{n+1}\right)$ и $v=\left(v_{1}, \ldots, v_{n+1}\right)$ из $X$ удовлетворяют условиям: $u_{i} \in U, v_{i} \in U, u_{j}=v_{j}$ для $j<i$, и $\rho_{j}\left(u_{j}, v_{j}\right)<\delta$ для $j>i$. В силу предположения теоремы 3 можно считать, что $U$ имеет в некоторой точке $x_{0 i} \in U(i, \delta)$-максимальное сечение. Возьмем произвольную окрестность $V$ точки $x_{i}$. Обозначим $S\left(x_{i}, V\right)=\sup F(y), I\left(x_{i}, V\right)=\inf F(z)$, где sup и $\inf$ берутся по точкам $y, z$ из $\varphi_{i}^{-1}(V)$. Положим $S\left(x_{i}\right)=\inf S\left(x_{i}, V\right)$ и $I\left(x_{i}\right)=\sup I\left(x_{i}, V\right)$, где $\inf$ и sup берутся по всем окрестностям $V$ точки $x_{i}$. Докажем, что

$$
S\left(x_{i}\right)-I\left(x_{i}\right) \leqslant 2 d .
$$

Возьмем в качестве $V$ внутренность $U_{0}$ шара $U$. Пусть точки $y=\left(y_{1}, \ldots, y_{n+1}\right)$ и $z=\left(z_{1}, \ldots, z_{n+1}\right)$ принадлежат $\varphi_{i}^{-1}\left(U_{0}\right)$. Тогда в $X$ найдутся точки $u=$ $\left(u_{1}, \ldots, u_{n+1}\right)$ и $v=\left(v_{1}, \ldots, v_{n+1}\right)$ такие, что $u_{i}=v_{i}=x_{0 i}, u_{j}=y_{j}, v_{j}=z_{j}$ для $j<i$ и и $\rho_{j}\left(u_{j}, y_{j}\right)<\delta, \rho_{j}\left(v_{j}, z_{j}\right)<\delta$ для $j>i$. Из (24) следуют неравенства $|h(u)-h(y)|<\varepsilon$ и $|h(v)-h(z)|<\varepsilon$. Так как $g(u)=g(y)$ и $g(v)=g(z)$, то $|F(u)-F(y)|<\varepsilon$ и $|F(v)-F(z)|<\varepsilon$. Из (23) следует $F(u)-F(v) \leqslant 2 d$, так как $\varphi_{i}(u)=\varphi_{i}(v)$. Тогда $F(y)-F(z)<2 d+2 \varepsilon$. Поэтому $S\left(x_{i}, U_{0}\right)-I\left(x_{i}, U_{0}\right) \leqslant 2 d+2 \varepsilon$. Отсюда $S\left(x_{i}\right)-I\left(x_{i}\right) \leqslant 2 d+2 \varepsilon$. Так как $\varepsilon$ сколь угодно мало, то верно неравенство (25). Следовательно, $S\left(x_{i}\right)-d \leqslant I\left(x_{i}\right)+d$.

Значения $S\left(x_{i}\right)$ и $I\left(x_{i}\right)$ определены для всех точек $x_{i} \in X_{i}$. Нетрудно проверить, что функция $S$ полунепрерьвна сверху, а функция $I$ полунепрерьвна снизу. По теореме Катетова о разделении полунепрерывных функций [11] существует непрерывная функция $g_{i}^{*}$ на $X_{i}$, удовлетворяюшая неравенствам $S\left(x_{i}\right)-d \leqslant g_{i}^{*}\left(x_{i}\right) \leqslant$ $I\left(x_{i}\right)+d$ для всех $x_{i} \in X_{i}$. Тогда $F(x)-d \leqslant g_{i}^{*}\left(x_{i}\right) \leqslant F(x)+d$. Это означает, что сумма $g_{1} \circ \varphi_{1}+\cdots+g_{i-1} \circ \varphi_{i-1}+g_{i}^{*} \circ \varphi_{i}+\cdots+g_{n}^{*} \circ \varphi_{n}$ есть наилучшее приближение функции $f$ в $B_{0}(X)$. По индукции получим наилучшее приближение $g_{1} \circ \varphi_{1}+g_{2}^{*} \circ \varphi_{2}+\cdots+g_{n}^{*} \circ \varphi_{n}$ для $f$ в $B_{0}(X)$, где функции $g_{2}^{*}, \ldots, g_{n}^{*}$ непрерывны. Обозначим теперь $F=f-g_{2}^{*} \circ \varphi_{2}-\cdots-g_{n}^{*} \circ \varphi_{n}$. В отличие от предыдущих вькладок функция $F$ на этот раз непрерывна. Поэтому замену $g_{1}$ на непрерывную функцию можно осушествить проше. Положим $S\left(x_{1}\right)=\sup F(y)$ и $I\left(x_{1}\right)=\inf F(z)$, где sup и inf берутся по точкам $y, z$ из сечения $\varphi_{1}^{-1}\left(x_{1}\right)$. Применяя доказательство от противного, легко убедиться, что функция $S$ полунепрерывна сверху, а функция I полунепрерывна снизу. Используя снова теорему Катетова, получим искомую непрерывную функцию $g_{1}^{*}$. Теорема доказана.

Приведем несколько простых примеров, иллюстрируюших теорему 3 . Пусть $X$ - конечное объединение параллелепипедов в $\mathbb{R}^{p}$, ребра которых параллельны координатным осям, $p \geqslant 2$. Положим $x_{1}=t_{1}, \ldots, x_{n}=t_{n}, x_{n+1}=\left(t_{n+1}, \ldots, t_{p}\right)$, $2 \leqslant n \leqslant p$, где $t_{1}, \ldots, t_{p}-$ координаты произвольной точки из $X$. При $n=p$ переменная $x_{n+1}$ отсутствует. Если $x_{i}-$ координата некоторой точки из $X, 2 \leqslant i \leqslant n$, то любая достаточно малая замкнутая окрестность точки $x_{i}$ в проекции множества $X$ на $i$-ю координатную ось имеет $i$-максимальное сечение в самой точке $x_{i}$. 
Очевидно, что $i$-максимальное сечение является также $(i, \delta)$-максимальньм сечением для той же окрестности при любом $\delta>0$. Поэтому предположение теоремы 3 выполняется. Пусть теперь $X$ - шар в пространстве $\mathbb{R}^{p}, p \geqslant 2$. Определим переменные $x_{1}, \ldots, x_{n}, x_{n+1}$ так же, как и в предыдушем примере. Если $x_{i}$-координата некоторой точки из $X, 2 \leqslant i \leqslant n$, то любая замкнутая окрестность точки $x_{i}$ имеет $i$-максимальное сечение в той точке этой окрестности, где $i$-я координата имеет наименьший модуль (предполагается, что координаты центра шара равны нулю). Поэтому предположение теоремы 3 снова вьполняется. Следующий пример показывает, что использование понятия $(i, \delta)$-максимального сечения вместо $i$-максимального сечения сушественно. Возьмем в качестве $X$ множество в координатном пространстве $O x_{1} x_{2} x_{3}$, заданное неравенствами $0 \leqslant x_{1} \leqslant 1,0 \leqslant x_{2} \leqslant 1$, $-3 \leqslant x_{1}+x_{2}+x_{3} \leqslant 3$. Если $x_{3}-$ координата точки из $X$, то любая замкнутая окрестность точки $x_{3}$ на оси $O x_{3}$ имеет 3 -максимальное сечение. Если $x_{2}$ - координата некоторой точки из $X$, то никакая замкнутая окрестность точки $x_{2}$ на оси $O x_{2}$ не имеет 2 -максимального сечения. Однако для любого $\delta>0$ найдется сколь угодно малая замкнутая окрестность точки $x_{2}$, которая имеет $(2, \delta)$-максимальное сечение в самой точке $x_{2}$. Свойства множества $X$ относительно координаты $x_{1}$ аналогичны. В этом примере предположение теоремы 3 выполняется как при $n=2$, так и при $n=3$.

Возможно, что условие теоремы 3 можно ослабить, но полностью его опустить нельзя, как показывает упомянутый во введении пример из работы [7]. В этом примере компактное множество $X$ на плоскости $O x y$ одномерно. Нетрудно построить пример с аналогичными свойствами, где $X$ является замкнутой ограниченной областью на плоскости $O x y$.

\section{Список литературы}

1. Diliberto S.P., Straus E. G. On the approximation of a function of several variables by the sum of functions of fewer variables // Pacif. J. Math. 1951. V. 1. № 1. P. 195-210.

2. Aumann G. Approximation by step functions // Proc. Amer. Math. Soc. 1963. V. 14. № 3. P. 477-482.

3. Медведев B. А. Опровержение одной теоремы Дилиберто и Страуса // Матем. заметки. 1992. Т. 51. № 4. С. 78-80.

4. Офман Ю. П. О наилучшем приближении функций двух переменных функциями вида $\varphi(x)+\psi(y) / /$ Изв. АН СССР. Сер. матем. 1961. Т. 25. № 2. С. 239-252.

5. Моторный В. П. К вопросу о наилучшем приближении функций двух переменных функциями вида $\varphi(x)+\psi(y) / /$ Изв. АН СССР. Сер. матем. 1963. Т. 27. №6. С. 1211-1214.

6. Моторный $B$. П. К вопросу о наилучшем приближении функций двух переменных функциями вида $\varphi(x)+\psi(y) / /$ Исследования по современным проблемам конструктивной теории функций. Баку: АН Аз. ССР, 1965. С. 66-72.

7. Хавинсон $C$. $A$. Чебышевская теорема для приближения функций двух переменных суммами $\varphi(x)+\psi(y) / /$ Изв. АН СССР. Сер. матем. 1969. Т. 33. № 4. С. 650-666.

8. Хавинсон C.Я. О представлении и приближении функций двух переменњых линейными суперпозициями // Вопросы математики, механики сплошных сред и применения математических методов в строительстве. М.: МИСИ, 1987. С. 89-92.

9. Арнольд В.И. О функциях трех переменных // ДАН СССР. 1957. Т. 114. № 4. C. $679-681$.

10. Дәй М. М. Линейные нормированные пространства. М.: ИЛ, 1961.

11. Katetov $M$. On real functions in topological spaces // Fund. Math. 1951. V. 38. P. 85-91. 